Tropical Journal of Pharmaceutical Research January 2020; 19 (1): 39-44

ISSN: $1596-5996$ (print); 1596-9827 (electronic)

(C) Pharmacotherapy Group, Faculty of Pharmacy, University of Benin, Benin City, 300001 Nigeria.

\title{
MiR-196b-5p regulates the proliferation of drug-resistant hepatocellular carcinoma cell lines by activating NFKB/ABCB1 signaling pathway
}

\author{
Bangming $\mathrm{Pu}^{1}$, Yong $\mathrm{CaO}^{2}$, Yan $\mathrm{Li}^{2}$, Li Tang ${ }^{2}$, Jiyi Xia ${ }^{3}$, Bo $\mathrm{Li}^{1 *}$ \\ ${ }^{1}$ Department of Hepatobiliary Surgery, ${ }^{2}$ Medicine Experimental Center, The Affiliated Hospital of South West Medical University, \\ ${ }^{3}$ School of Medical Information and Engineering, Southwest Medical University, Luzhou 646000, China
}

*For correspondence: Email: liboer2012@163.com; Tel: +86-830-3160048

Sent for review: 24 August 2019

Revised accepted: 20 December 2019

\begin{abstract}
Purpose: To explore the molecular function of miR-196b-5p in hepatocellular carcinoma (HCC).

Methods: MiR-1966-5p expression levels in HCC tissue samples were assessed by qRT-PCR. MiR1966-5p was knocked-down or over-expressed in HepG2 cells by transfecting the cells with plasmids expressing either a miR-196b-5p inhibitor or mimic, respectively, while cell proliferation was assessed by MTT assay. The interaction of miR-196b-5p with target molecules was confirmed using luciferase reporter assay. Cell cycle was investigated by flow cytometry, while NFKBIA expression was assessed by western blotting.

Results: MiR-196b-5p was over-expressed in HCC, and miR-196b-5p expression levels in patients with HCC were related to tumor grade. MiR-196b-5p over-expression promoted cell proliferation and colony formation and suppressed cell cycle arrest and apoptosis. The results of luciferase reporter assay showed that miR-196b-5p reduced NFKBIA expression in HepG2 cells by binding to a response element in the 3' UTR of NFKBIA. Further investigation showed that NFKBIA interacts with NFKB1 and reduces the concentration of NFKB1 in HepG2 cells. The promoter of ATP-binding cassette sub-family $B$ member 1 (ABCB1) was also targeted and bound by NFKB1, which altered the expression of ABCB1 in HepG2 cells.

Conclusion: MiR-196b-5p regulates cell proliferation in drug-resistant HCC cell lines via activation of the NFKB/ABCB1 signaling pathway.
\end{abstract}

Keywords: Hepatocellular carcinoma, miR-196b-5p, NFKBIA, NFKB1, ABCB1

\begin{abstract}
This is an Open Access article that uses a fund-ing model which does not charge readers or their institutions for access and distributed under the terms of the Creative Commons Attribution License (http://creativecommons.org/licenses/by/4.0) and the Budapest Open Access Initiative (http://www.budapestopenaccessinitiative.org/read), which permit unrestricted use, distribution, and reproduction in any medium, provided the original work is properly credited.

Tropical Journal of Pharmaceutical Research is indexed by Science Citation Index (SciSearch), Scopus, International Pharmaceutical Abstract, Chemical Abstracts, Embase, Index Copernicus, EBSCO, African Index Medicus, JournalSeek, Journal Citation Reports/Science Edition, Directory of Open Access Journals (DOAJ), African Journal Online, Bioline International, Open-J-Gate and Pharmacy Abstracts
\end{abstract}

\section{INTRODUCTION}

Approximately $90 \%$ of primary liver cancers are hepatocellular carcinoma (HCC). The morbidity of HCC is among the highest of all cancers [1], and many cases of $\mathrm{HCC}(50 \%)$ are diagnosed at an advanced stage [2]. Although various treatments have been developed for HCC, the prognosis is still poor [3,4].

HCC is a highly angiogenic, solid tumor that is mainly induced by dysregulation of angiogenesis and cell proliferation and evasion of apoptosis. Multiple genetic and epigenetic changes, 
chromosomal aberrations, and molecular pathway alterations have been shown to be involved in the molecular pathogenesis of $\mathrm{HCC}$ [5].

Micro RNAs (miRNAs) are a group of small (1825 nucleotides), non-coding RNAs [6] that modulate gene expression by binding to the $3^{\prime}$ untranslated region (UTR) of target messenger RNAs (mRNAs). This binding can result in either degradation of the mRNA or suppression of its expression [7]. Aberrant expression of miRNAs has been reported to be useful for diagnosis, classification, and prognosis prediction of various cancers [8], and recent studies have shown that multiple miRNAs are involved in the tumorigenesis of HCC [9]. Targeting these miRNAs may offer novel therapeutic strategies for the treatment of HCC [10].

MiR-196b-5p has been implicated in several diseases, including prostate and liver cancer $[11,12]$, and it has been shown to be involved in the regulation of cell proliferation, metastasis, and chemoresistance. However, the function of miR-196b-5p in HCC has not been explored.

Thus, in the present study, the molecular function of miR-196b-5p in HCC was investigated. Determining the physiological function of miR$196 b-5 p$ in HCC may reveal its potential as a novel biomarker or therapeutic target.

\section{EXPERIMENTAL}

\section{Clinical samples}

HCC tumor samples $(n=116)$ and normal liver tissue samples $(n=47)$ were collected from patients who underwent liver excision at the Affiliated Hospital of Southwest Medical University from January 2015 to May 2017. The collected tissues were snap-frozen after surgery and stored at $-80^{\circ} \mathrm{C}$ until use. The use of human tissues in this investigation was approved by The Affiliated Hospital of South West Medical University (approval no. AHSWMU-2017-121). Experiments using human tissues were carried out in accordance with international ethical guidelines for biomedical research involving human subjects [13]. All study subjects provided consent for their participation in the study.

\section{Cell culture}

The normal hepatocyte cell line LO2 and the HCC drug-resistant cell lines Hep3B, MHCC97H, HepG2, HCC9204, SNU-398, and HepaRG were obtained from the Global Bioresource
Center (USA). All cells were cultured in DMEM containing $10 \%$ fetal bovine serum.

\section{Cell transfection}

HepG2 cells were transfected with a miR-196b$5 p$ inhibitor, miR-196b-5p mimic, or empty vector (GenScript) using Lipofectamine 2000 (Invitrogen; Thermo Fisher Scientific, Waltham, MA, USA) and Opti-MEM reagents (Gibco; Thermo Fisher Scientific). The cells were also transfected with pcDNA-NFkBIA (NFKBIA)/NFkBIA-siRNA (NFKBIA-RNAi) or pcDNA-NFKB1 (NFKB1)/NFKB1-siRNA (NFKB1RNAi).

\section{qRT-PCR}

Total RNA was isolated from clinical tissue samples using TRIzol reagent (Thermo Fisher). Then, $2 \mu \mathrm{g}$ of the isolated total RNA was used to synthesize cDNA with the PrimeScript kit (Thermo Fisher). PCR was performed with the TransStart Tip Green qPCR SuperMix kit (GenScript). The $2^{-\Delta \Delta C t}$ method was used to compare the relative expression levels of target genes.

\section{MTT assay}

Cells were seeded into plates (Corning, Corelle City, NY, USA) and incubated at $37^{\circ} \mathrm{C}$ for $0,1,2$, 3 , and 4 days. Then, $10 \mu \mathrm{L}$ of $5 \mathrm{mg} / \mathrm{mL}$ MTT solution (Thermo Fisher) was added to the medium and incubated for $4 \mathrm{~h}$. After incubation, $\sim 200 \mu \mathrm{L}$ of formazan in dimethylsulfoxide (DMSO; Genview, Beijing, China) was added. Finally, absorbance (A) at $490 \mathrm{~nm}$ was recorded with a microplate reader (BMG LABTECH). The rate of suppression (S) was calculated in Eq 1.

$S=\left(A_{\text {control }}-A_{\text {treatment }}\right) / A_{\text {control }}$.

\section{Colony formation}

Cells were cultured in DMEM for 14 days. Then, $4 \%$ paraformaldehyde was added to the culture medium, and the cells were incubated for $1 \mathrm{~h}$. Next, the cells were stained with Giemsa (Thermo Fisher) for $15 \mathrm{~min}$, and the number of colonies was counted under an inverted microscope (Olympus, Tokyo, Japan).

\section{Flow cytometry}

Transfected HepG2 cells were incubated for 48 $\mathrm{h}$, fixed with $60 \%$ ethanol, and incubated at $4^{\circ} \mathrm{C}$ for $12 \mathrm{~h}$. Then, the cells were collected by centrifugation and resuspended in PBS. DNA content was assessed by using a DNA Prep 
Stain kit (Thermo Fisher), and the proportions of cells in G0/G1, S, and G2/M phases were determined by an Attune NxT flow cytometer (Thermo Fisher).

\section{Western blotting}

HepG2 cells were collected and lysed in RIPA buffer (Thermo Fisher) to generate crude extracts, and then the proteins in the extracts were separated by $15 \%$ SDS-PAGE. Subsequently, the separated proteins were transferred to PVDF membranes (Thermo Fisher Scientific) by semi-dry transfer. The membranes were blocked by incubation with $5-10 \%$ BSA for $1-2 \mathrm{~h}$. Then, the membranes were incubated with primary antibodies against NFKBIA and $\beta$-actin (Abcam,Cambridge, MA, USA) at $25^{\circ} \mathrm{C}$ for $2 \mathrm{~h}$.

After washing, the blot was incubated with a horseradish peroxidase-conjugated secondary antibody $\left(1: 3000\right.$, Abcam) at $25^{\circ} \mathrm{C}$ for $1-2 \mathrm{~h}$. Finally, the blots were incubated with $\mathrm{ECL}$ reagent (Amersham Pharmacia Biotech, Amersham, UK) and visualized with an Amersham Imager 600 (GE).

\section{Luciferase reporter assay}

Wild type and mutant NFKBIA 3' UTR sequences (NFKBIA-WT and NFKBIA-MUT, respectively) were cloned into the pGL3 luciferase reporter plasmid (Thermo Fisher) to generate pGL3NFKBIA-WT and pGL3-NFKBIA-MUT, respectively. HepG2 cells $\left(4.5 \times 10^{5}\right.$ cells/well $)$ were co-transfected with either pGL3-NFKBIAWT or pGL3-NFKBIA-MUT and either a miR196b-5p mimic, miR-196b-5p inhibitor, or empty vector using Lipofectamine 2000 (Invitrogen).

The NFKB1 and ABCB1 promoters were also cloned into pGL3 (Promega) to generate pGL3NFKB1 and pGL3-ABCB1, respectively. HepG2 cells $\left(5 \times 10^{5}\right.$ cells/well) were co-transfected with either pGL3-NFKB1 or pGL3-ABCB1 and either an NFKBIA-overexpression vector or NFKBIARNAi using Lipofectamine 2000 (Invitrogen). After a 48-h incubation period, luciferase activity was assessed using the dual luciferase reporter assay kit (Invitrogen).

\section{Statistical analysis}

Data are shown as the mean \pm standard deviation (SD). GraphPad was used to analyze the data. Student's t-test and one-way analysis of variance (ANOVA) were used to compare groups. Differences with $p<0.05$ were considered statistically significant.

\section{RESULTS}

\section{MiR-196b-5p is highly expressed in HCC}

MiR-196b-5p levels in clinical HCC tissues and cells were evaluated by QT-PCR. MiR-196b-5p levels were significantly higher in HCC tissues than in normal tissues $(p<0.05$; Figure $1 \mathrm{~A})$, and miR-196b-5p levels were positively related to HCC tumor grade (Figure $1 \mathrm{~B}$ ). In addition, miR196b-5p was up-regulated in HepG2 cells (Figure 1C). MiR-196b-5p levels were also assessed in the L-O2 normal hepatocyte cell line as well as several HCC cell lines, including Hep3B, MHCC97-H, HCC9204, SNU-398, and HepaRG. As shown in Figure $1 \mathrm{C}$, miR-196b-5p was significantly over-expressed in the HCC cell lines when compared to the levels in L-O2 cells $(p<$ 0.05).

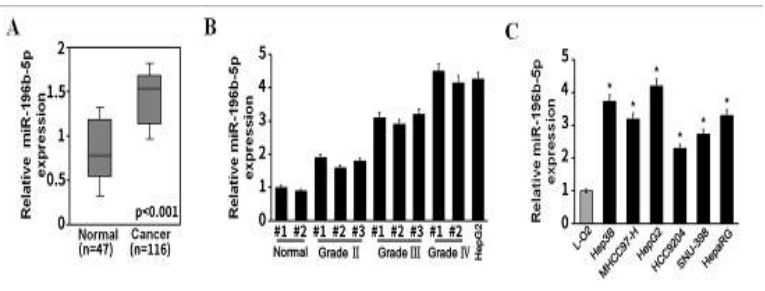

Figure 1: MiR-196b-5p was significantly up-regulated in HCC. A. MiR-196b-5p levels in HCC tumors ( $n=$ 116) and normal liver tissues $(n=47)$ were evaluated by QT-PCR ( ${ }^{*} p<0.05$ vs. Normal). B. MiR-196b-5p levels in patients with different grades of HCC was assessed by QT-PCR. C. MiR-196b-5p levels in normal L-O2 hepatocytes and Hep3B, MHCC97-H, HepG2, HCC9204, SNU-398, and HepaRG HCC cells were determined by QT-PCR ( ${ }^{*} p<0.05$ vs. L-O2)

\section{MiR-196b-5p regulates the pathological functions of HepG2 cells}

To further evaluate the role of miR-196b-5p in HCC, HepG2 cells were transfected with a miR196b-5p mimic, a miR-196b-5p inhibitor, or empty vector. MiR-196b-5p levels in HepG2 cells were dramatically increased after transfection with a miR-196b-5p mimic (Figure $2 \mathrm{~A}, p<0.05$ vs. vector), but were greatly down-regulated by the introduction of a miR-196b-5p inhibitor $(p<$ 0.05 vs. vector). Figure $2 \mathrm{~B}$ shows that cell proliferation was markedly promoted by transfection of a miR-196b-5p mimic but was strongly inhibited by transfection of a miR-196b$5 p$ inhibitor ( $p<0.05$ vs. vector). Furthermore, transfection of a miR-196b-5p mimic led to the formation of a significantly greater number of colonies, while transfection of a miR-196b-5p inhibitor reduced colony numbers ( $p<0.05$ vs. vector; Figure $2 \mathrm{C}$ ). As shown in Figure $2 \mathrm{D}$, the proportion of cells in G0/G1 phase was increased 
in HepG2 cells transfected with a miR-196b-5p mimic, while transfection of a miR-196b-5p inhibitor decreased the proportion of cells in $\mathrm{G} 0 / \mathrm{G} 1$ phase ( $p<0.05$ vs. vector).
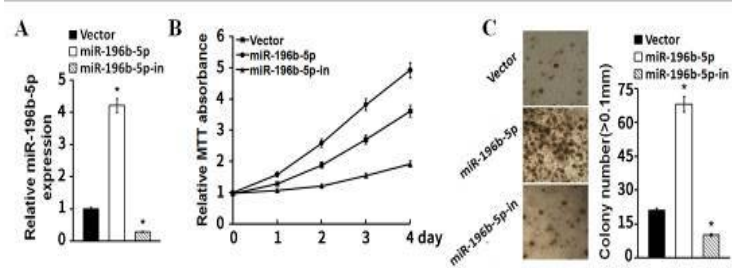

D

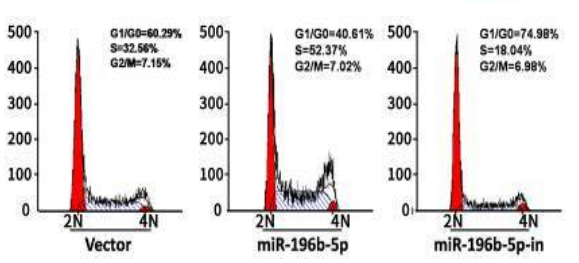

Figure 2: Impact of miR-196b-5p on the pathological functions of HepG2 cells. A. MiR-196b-5p levels in miR-196b-5p mimic- and miR-196b-5p inhibitortransfected HepG2 cells was assessed by QT-PCR. B. Cell proliferation in these transfected cells was evaluated by the MTT assay. C. Colony formation was also assayed in these transfected cells. D. Flow cytometry was performed to assess the cell cycle status of the cells ( $p<0.05$ vs. Vector)

\section{MiR-196b-5p directly targets NFkBIA in HepG2 cells}

To identify the target genes of miR-196b-5p that may be related to HCC, qRT-PCR was performed to determine the expression levels of tumorigenesis-associated genes in miR-196b-5p mimic- and miR-196b-5p inhibitor-transfected HepG2 cells. As shown in Figure $3 \mathrm{~A}$, the expression levels of CCND1, Oct4, CD44, MMP9, Twist, LEF1, Nanog, Tiam1, and AXIN2 were increased following transfection of miR196b-5p mimic and decreased following transfection of miR-196b-5p inhibitor. In addition, the expression levels of FASL, Bim, p21, p27, Bax, and caspase-3 were deceased in miR196b-5p mimic-transfected HepG2 cells and upregulated in miR-196b-5p inhibitor-transfected HepG2 cells (Figure $3 \mathrm{~B}$ ).

To further investigate the molecular mechanism underlying the effects of miR-196b-5p in HCC, TargetScan, an online target prediction program, was utilized to identify additional target genes of miR-196b-5p. The results identified NFkBIA as a target gene of interest (Figure $3 \mathrm{C}$ ). qRT-PCR showed that NFKBIA expression was significantly suppressed following transfection of a miR-196b$5 p$ mimic but was promoted following transfection of a miR-196b-5p inhibitor ( $p<0.05$ vs. vector; Figure $3 \mathrm{D}$ ). As shown in Figure $3 \mathrm{E}$ and $\mathrm{F}$, luciferase activity was increased in cells transfected with both pGL3-NFKBIA-WT and miR-196b-5p inhibitor and was markedly decreased in cells transfected with both pGL3NFkBIA-WT and miR-196b-5p mimic.

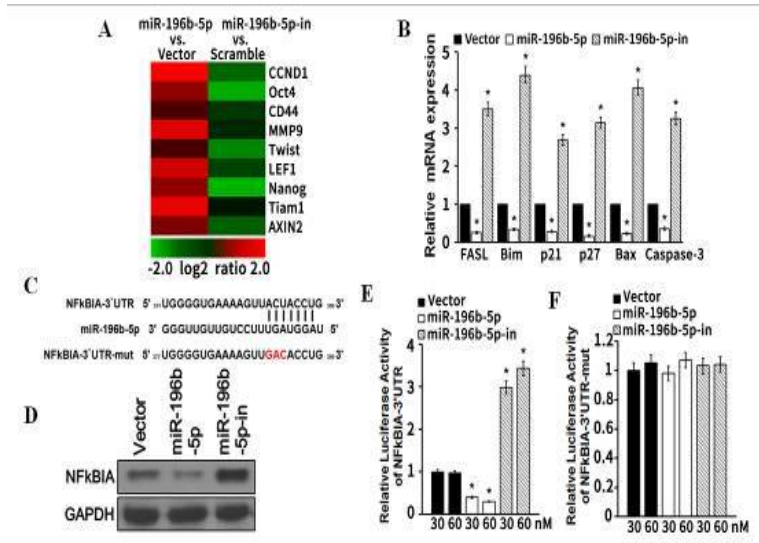

Figure 3: MiR-196b-5p directly targets NFKBIA in HepG2 cells. A. The expression levels of tumorigenesis-related genes was evaluated by qRTPCR. B. Relative mRNA levels of FASL, Bim, p21, p27, Bax, and caspase-3 were determined by qRTPCR. C. Online prediction of miR-196b-5p binding sites near NFkBIA. D. NFkBIA levels in HepG2 cells transfected with miR-196b-5p mimic, miR-196b-5p inhibitor, or empty vector were evaluated by western blotting. E. Relative luciferase activity in HepG2 cells co-transfected with pGL3-NFKBIA-WT luciferase reporter and either miR-196b-5p mimic, miR-196b-5p inhibitor, or empty vector. F. Relative luciferase activity HepG2 cells co-transfected with pGL3-NFkBIA-mut luciferase reporter and either miR-196b-5p mimic, miR-196b-5p inhibitor, or empty vector $\left({ }^{*} p<0.05\right.$ vs. vector)

\section{NFKBIA directly regulates the NFKB1/ABCB1 axis in HepG2 cells}

As shown in Figure $4 \mathrm{~A}$, NFKB1 reporter activity was markedly decreased in NFKBIAoverexpression vector-transfected cells and increased in NFKBIA-RNAi-transfected cells. Furthermore, ABCB1 expression was induced by NFKB1 overexpression and suppressed by NFkB1 knock-down (Figure 4 B). Luciferase activity in HepG2 cells was increased following transfection with an NFKB1-overexpression vector but was reduced following transfection with NFkB1-RNAi (Figure $4 \mathrm{C}-\mathrm{E}$ ). Moreover, $A B C B 1$ expression was greatly increased in $\mathrm{HCC}$ tumors (Figure 4F).

\section{DISCUSSION}

It has been reported that miR-196b-5p is highly expressed in colorectal cancer tumors and that up-regulation of miR-196b-5p in colorectal cancer is associated with a lower survival rate [11]. 


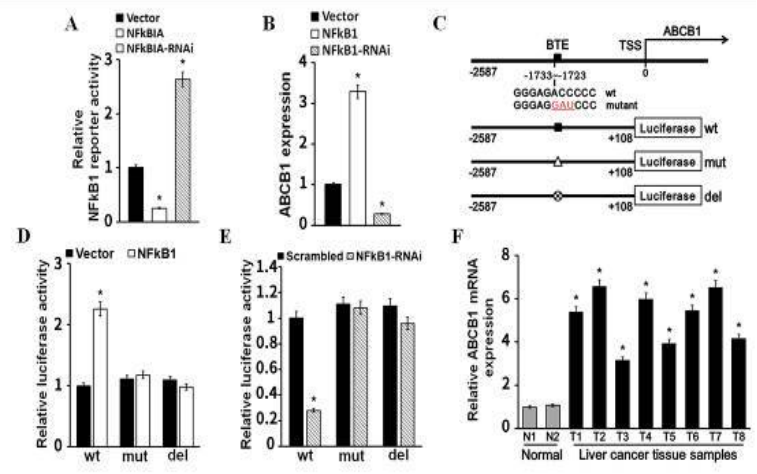

Figure 4: NFKBIA directly regulates the NFkB1/ABCB1 axis in HepG2 cells. A. The relationship between NFKB1 and NFKBIA was evaluated by a luciferase reporter assay. B. ABCB1 expression levels in transfected HepG2 cells were determined by qRT-PCR ( $p<0.05$ vs. vector). C-E. The relationship between NFKB1 and ABCB1 was evaluated by a luciferase reporter assay $(p<0.05$ vs. vector). F. ABCB1 expression levels in HCC tumors and healthy liver tissues were compared $(p<0.05$ vs. normal)

Further investigation showed that miR-196b-5p promoted the stemness of colorectal tumor cells by targeting suppressor of cytokine signaling (SOCS)-1 and SOCS3, which inhibit the STAT3 signaling pathway. Additionally, drug resistance in colorectal tumor cells was promoted by introduction of miR-196b-5p, and the sensitivity of colorectal cancer cells to chemotherapeutics was increased by knocking down miR-196b-5p [11]. These results indicate that the progression of colorectal cancer could be promoted by miR196b-5p.

Zhu et al [12] reported down-regulation of miR196b-5p in breast cancer tumors, and lymph node metastasis and clinical stage in breast cancer patients were also reported to be related to miR-196b-5p expression levels. Transfection of miR-196b-5p greatly suppressed the propagation ability of breast tumor cells by regulating collagen type I alpha 1 (COL1A1) [12]. These findings indicated that miR-196b-5p exerts tumor-inhibiting effects in breast cancer. In present study, miR-196b-5p was found to be highly over-expressed in HCC, and up-regulation of miR-196b-5p, by transfection of miR-196b-5p mimic, promoted the proliferation and colony formation of HepG2 hepatocyte cells and greatly inhibited cell cycle progression and apoptosis.

$\mathrm{NF} \kappa \mathrm{B}$ has been reported to play a role in multiple physiological processes, such as immune responses, cell adhesion, differentiation, proliferation, cell survival, and cytokine production $[14,15]$. The main members of the
NFkB family are P52/p100, p50/p105, c-Rel, RelB, and p50/p105 [16]. Under normal physiological conditions, NFKB is located in the cytoplasm, and its activity is inhibited via sequestration by proteins such as NFKB inhibitor (IKB)a, $\beta$, or $\gamma$ and NFKBIA [17]. NFKB1, which encodes the NFKB p105 subunit, is a downstream gene of NFKBIA.

It has been demonstrated that low expression levels of NFKB1 and NFKBIA are related to the tumorigenesis of colorectal cancer. Song et al [18] reported that polymorphisms of NFKB1 and NFKBIA are a major factor inducing colorectal cancer. In the present study, we demonstrated that miR-196b-5p directly targets NFKBIA in HepG2 cells and thereby regulates the activity of NFKB1. Our results indicated that introduction of miR-196b-5p activated the NFKB signaling pathway in HepG2 cells by regulating the activity of NFKB1 and NFKBIA. ABCB1 encodes Pglycoprotein 1 (P-gp), which functions as a foreign substances pump [19] and has been implicated in drug resistance in various cancers [20-22]. The present results demonstrated that NFkB1 interacts with ABCB1 in HepG2 cells. Moreover, ABCB1 levels were greatly increased in HCC tumors. These results also imply that miR-196b-5p regulates cell propagation, colony development, the cell cycle, and apoptosis by regulating $A B C B 1$ expression through the NFKB signaling pathway.

\section{CONCLUSION}

The findings of this study reveal that miR-196b$5 p$ expression is significantly upregulated in HCC tissues and that miR-196b-5p expression is associated with tumor grade in HCC patients. Overexpression of miR-196b-5p in HCC cell lines induces cell proliferation and colony formation, and suppresses cell cycle arrest and cell apoptosis. MiR-196b-5p exerts its pro-tumor roles via NFKB/ABCB1 signaling in HCC cell lines. Thus, these findings suggest that miR$196 b-5 p$ is a potential therapeutic target as well as biomarker for HCC.

\section{DECLARATIONS}

\section{Acknowledgement}

The work was supported by the Luzhou Science and Technology Bureau (Project no. 2014-S-44), Sichuan Education Department (Project no. 16ZA089), Sichuan Traditional Chinese Medicine Administration (Project no. 2018JC029), and Luzhou Science and Technology Bureau- 
Southwest Medical University (Joint Project no. 2015LZCYD-S01[2/15]).

\section{Conflict of interest}

No conflict of interest is associated with this work.

\section{Contribution of authors}

We declare that this work was done by the authors named in this article and all liabilities pertaining to claims relating to the content of this article will be borne by the authors.

\section{Open Access}

This is an Open Access article that uses a funding model which does not charge readers or their institutions for access and distributed under the terms of the Creative Commons Attribution License (http://creativecommons.org/licenses/by/ 4.0) and the Budapest Open Access Initiative (http://www.budapestopenaccessinitiative.org/rea d), which permit unrestricted use, distribution, and reproduction in any medium, provided the original work is properly credited.

\section{REFERENCES}

1. Elserag HB. Hepatocellular carcinoma: epidemiology and molecular carcinogenesis. Gastroenterol 2007; 132: 2557-2576.

2. Stefano, Colagrande, Andrea, Inghilesi, Aburas, Gian, Taliani, Cosimo, Nardi. challenges of advanced hepatocellular carcinoma. World J Gastroenterol 2016; 22: 7645-7659.

3. Schlachterman A, Jr WWC, Hilgenfeldt $E$, Mitra $A$, Cabrera R. Current and future treatments for hepatocellular carcinoma. World J Gastroenterol 2015; 21: 8478-8491.

4. Bruix J, Sherman M. Management of hepatocellular carcinoma: An update. Hepatol 2011; 53: 1020.

5. Farazi $P A$, Depinho RA. Hepatocellular carcinoma pathogenesis: from genes to environment. Nat Rev Cancer 2006; 6: 674-687.

6. Bartel DP. MicroRNAs. Cell 2004; 116: 0-297.

7. Ersonbensan $A E$. Introduction to microRNAs in biological systems. Methods Mol Biol 2014; 1107: 1-14.
8. Garzon R, Croce CM. MicroRNAs and Cancer: Introduction. Seminars in Oncol 2011; 38: 721-723.

9. Yang S, Fei W, Qi H, Yan C, Yingying Z, Changqing Y. MicroRNAs Contribute to Hepatocellular Carcinoma. Mini-Rev Med Chem 2015; 15: 459-466.

10. Hayes CN, Chayama K. MicroRNAs as Biomarkers for Liver Disease and Hepatocellular Carcinoma. Int J Mol Sci 2016; 17: 280.

11. Ren $D$, Lin $B$, Zhang $X$, Peng $Y, Y e Z$, Ma $Y$, Liang $Y$, Cao L, Li X, Li R. Maintenance of cancer stemness by miR-196b-5p contributes to chemoresistance of colorectal cancer cells via activating STAT3 signaling pathway. Oncotarget 2017; 8: 49807-49823.

12. Zhu X, Rao X, Yao W, Zou X. Downregulation of MiR$196 b-5 p$ impedes cell proliferation and metastasis in breast cancer through regulating COL1A1. Am J Transl Res 2018; 10: 3122-3132

13. S. Council for International Organizations of Medical, International ethical guidelines for biomedical research involving human subjects, Bull Med Ethics (2002) 17-23.

14. Baldwin AS. Series introduction: the transcription factor NF-kappaB and human disease. J Clin Invest 2001; 107: 3-6.

15. Gilmore T. Introduction to NF-kappaB: players, pathways, perspectives. Oncogene 2006; 25: 6680-6684.

16. Blank V, Kourilsky $P$, Israël $A$. NF-KB and related proteins: Rel/dorsal homologies meet ankyrin-like repeats. Trends Biochem Sci 1992; 17: 135.

17. Hayden MS, West AP, Ghosh S. SnapShot: NF-kappaB signaling pathways. Cell 2006; 127: 1286-1287.

18. Song S, Chen D, Lu J, Liao J, Luo Y, Yang Z, Fu X, Fan $X$, Wei $Y$, Yang L. NFKB1 and NFKBIA polymorphisms are associated with increased risk for sporadic colorectal cancer in a southern Chinese population. Plos One 2011; 6: e21726.

19. Wolking S, Schaeffeler E, Lerche H, Schwab M, Nies AT. Impact of Genetic Polymorphisms of ABCB1 (MDR1, PGlycoprotein) on Drug Disposition and Potential Clinical Implications: Update of the Literature. Clin Pharmacokine 2015; 54: 709-735.

20. Tulsyan S, Mittal RD, Mittal B. The effect of ABCB1 polymorphisms on the outcome of breast cancer treatment. Pharmgenomics Pers Med 2016; 9: 47-58.

21. Coyle B, Kessler M, Sabnis $D H$, Kerr ID. ABCB1 in children's brain tumours. Biochem Soc Trans 2015; 43: 1018-1022.

22. Maia RC, Vasconcelos FC, Souza PS, Rumjanek VM. Towards Comprehension of the ABCB1/P-Glycoprotein Role in Chronic Myeloid Leukemia. Molecules 2018; 23: 119. 